\title{
Control of an omnidirectional mobile robot with wheels connected by passive sliding joints
}

\author{
Tatsuro TERAKAWA*, Masaharu KOMORI* and Kenji FUJIMOTO** \\ *Department of Mechanical Engineering and Science, Kyoto University \\ Kyoto daigaku-katsura, Nishikyo-ku, Kyoto, 615-8540, Japan \\ E-mail: komorim@me.kyoto-u.ac.jp \\ ${ }^{* *}$ Department of Aeronautics and Astronautics, Kyoto University \\ Kyoto daigaku-katsura, Nishikyo-ku, Kyoto, 615-8540, Japan
}

Received: 4 July 2018; Revised: 17 October 2018; Accepted: 20 December 2018

\begin{abstract}
To solve problems of conventional mobile robots, such as constrained mobility due to nonholonomic wheels or complicated structure due to specialized wheels, we propose a novel omnidirectional mobile robot named slidable-wheeled omnidirectional mobile robot (SWOM). SWOM has three conventional wheels connected to the main body by passive sliding joints, which enable the main body to make omnidirectional movement in spite of the nonholonomic constraints on the wheels. Thus, SWOM achieves both superb mobility and simple structure. However, its behavior is described as a nonlinear system with nonholonomic constraints, which has difficulty with control because this type of system does not have a general design method for a stabilizing controller. This study aims to develop a controller for SWOM using state feedback linearization. This paper presents the following achievements: An exactly linearized state equation is derived using feedback and coordinate transformation based on the kinematics of SWOM. The unwanted singular configurations of the system are discussed and a control strategy to avoid them is proposed. Then, a stabilizing controller that enables SWOM to reach a designated position and orientation is designed. Through a numerical simulation and an experiment using a SWOM prototype, the effectiveness of the developed control system was verified.
\end{abstract}

Keywords : Omnidirectional mobile robot, Wheel, Nonholonomic constraint, Feedback linearization, Singular configuration

\section{Introduction}

Mobile robots are now capable of working in a variety of fields, including industry and logistics (Cardarelli et al., 2017; Causo et al., 2017; Nielsen et al., 2017), welfare and nursing care (Gross et al., 2017; Saegusa et al., 2010; Tobita et al., 2018), and agriculture and forestry (Masuzawa et al., 2017; Sori et al., 2018; Tanaka et al., 2017). Conventional mobile robots utilize wheels driven in one direction by a motor or wheels steerable around the ground contact point. Such wheeled mechanisms have the advantages of simple structure and high durability. However, they can only travel in one direction and turn, so mobile robots with these wheels need switchbacks or turnabouts to move sideways. Such movements cost extra time and space, and these robots have difficulty in moving smoothly in environments with narrow passages or many obstacles.

Omnidirectional mobile robots, which can immediately move in arbitrary directions without switchbacks or turnabouts, have been proposed to address this issue. A primary example is an omnidirectional mobile robot with omni or Mecanum wheels, which have freely rotating rollers installed around the perimeter of the wheel (Campion et al., 1990; Muir and Neuman, 1987). Their superb mobility is useful for competition robots (de Best et al., 2011; Samani et al., 2004) and carriers of mobile manipulators (Bischoff et al., 2011; Guo et al., 2016). An active omni wheel, which actively drives the rollers, has also been proposed (Komori et al., 2016). However, these wheels have the disadvantages such as low load capacity due to their complicated structure, unavoidable vertical vibration due to discontinuous ground contact, and inability to travel over tall obstacles due to the small radius of the rollers (Ferrière et al., 1996). As 


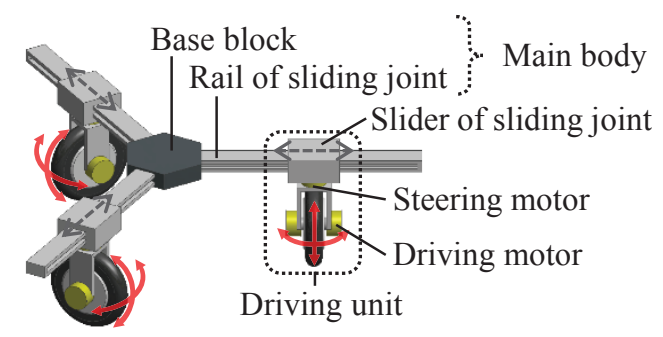

Fig. 1 SWOM proposed by Terakawa et al. (2018)

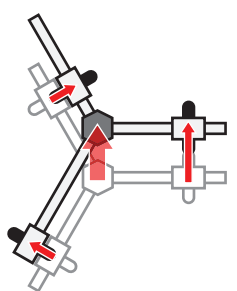

(a)

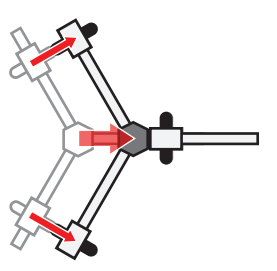

(b)

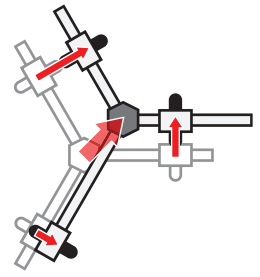

(c)

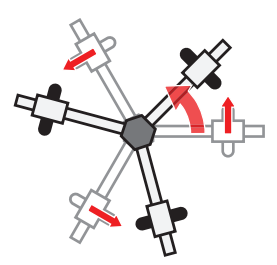

(d)

Fig. 2 Omnidirectional movement of SWOM using the relative movements of the sliding joints: (a) Forward translation. (b) Right translation. (c) Diagonal translation. (d) Turning. The upward direction in the figure is the forward direction.

another example, omnidirectional mobile robots using spherical wheels have been proposed (Kumagai and Ochiai 2010; Ok et al., 2011; Ostrovskaya et al., 2000; Singh et al., 2017; Wada and Asada, 1999; West and Asada, 1995). They also realize superb mobility but have problems in that the drive mechanisms tend to be complicated and the wheel radii become small despite their large volume.

To solve these problems, some of the authors have proposed a novel omnidirectional mobile robot - the slidable-wheeled omnidirectional mobile robot (SWOM) (Terakawa et al., 2018). SWOM has three actively driven and steerable wheels connected to the main body with passive sliding joints. These sliding joints enable SWOM to make omnidirectional movement. The wheels of SWOM are all conventional wheels, unlike the other omnidirectional mobile robots described above, so SWOM is free from the problems caused by a complicated wheel structure. Thus, SWOM can realize both superb mobility and simple structure.

As a next step, we needed to construct a control system for SWOM because it was not developed in our previous research. The description of its behavior includes nonlinearity resulting from the sliding joints and nonholonomic constraints of the wheels. This type of system does not have a generally available control method, so designing a controller for the system is difficult. To deal with this task, we intend to design a controller for SWOM using feedback linearization, which has been used to control conventional wheeled mobile robots with nonholonomic constraints (d'Andrea-Novel et al., 1992 and 1995). In this paper, we derive the exactly linearized state equation of SWOM using feedback and coordinate transformation based on a kinematic model, and then design a stabilizing controller for SWOM considering singular configurations. A numerical simulation and an experiment are conducted to verify its effectiveness.

\section{Structure and motion of SWOM}

First, we explain the structure of SWOM. As shown in Fig. 1, SWOM mainly consists of a base block, three sliding joints, and three driving units. The base block connects three rails of the sliding joints radially at equal intervals. The base block and three rails are the main body. Each driving unit has a slider of the sliding joint, a wheel, a driving motor, and a steering motor. The driving motor rotates the wheel around its axle, whereas the steering motor rotates the wheel around the ground contact point. There are no offsets between the axle and the ground contact point of the wheel. The sliding joint is a passive joint that moves depending on the driving unit movement, which changes the distance between the base block and the driving unit.

Next, we explain the omnidirectional movement of SWOM. Figure 2 illustrates examples of SWOM motion. In this figure, although the traveling direction of each wheel is always perpendicular to the rail of the sliding joint, SWOM 


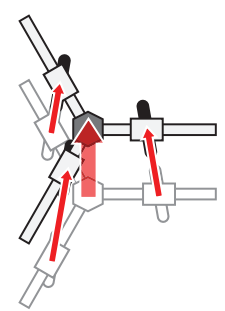

(a)

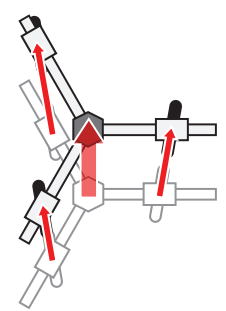

(b)

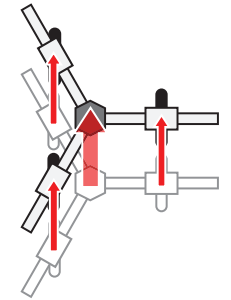

(c)

Fig. 3 Differences in the relative movements of the sliding joints depending on the steering angle of each wheel when SWOM is moving forward (toward the top of the figure): (a) Inward slider displacement. (b) Outward slider displacement. (c) No slider displacement.

moves in the forward, right, and diagonal directions and turns. The driving unit using a normal wheel can move only in the traveling direction of the wheel; it can turn, but it cannot move sideways. However, the main body can move not only in the moving direction of the driving units but also sideways of the driving units using the relative movements of the sliding joints. This means that the main body can achieve planar motion with three degrees of freedom (DOF) by rotating the wheels at appropriate velocities. Additionally, the moving direction of the main body can change immediately without changing the directions of the wheels because the steering angle of each wheel is constant in every movement shown in Fig. 2. Thus, SWOM can make omnidirectional movement.

Figure 2 shows the case where the traveling direction of each wheel is perpendicular to the rail, but SWOM is capable of omnidirectional movement with the wheels steered in other directions. The DOF of steering angles of wheels enables SWOM to manipulate the relative movement of the sliding joints in addition to the motion of the main body. We explain the case where SWOM is moving forward in Fig. 3. As shown in Fig. 3(a), when the traveling directions of the wheels are set inward to the moving direction of the main body (i.e., forward direction), the sliding joints move toward the base block while the main body moves forward. In contrast, when the traveling directions of the wheels are set outward, as shown in Fig. 3(b), the sliding joints move away from the base block. When the traveling directions of the wheels agree with the moving direction of the main body, as shown in Fig. 3(c), the main body moves but the sliding joints do not. Thus, SWOM can control the relative position of the sliding joints by steering each wheel appropriately while the main body moves along the desired trajectory. When the relative positions of the sliding joints are controlled within a certain range, SWOM can move without restriction, even with finite rail lengths, though infinite rail lengths are required if SWOM continues moving with the fixed steering angles of the wheels as in Fig. 2.

With respect to the steering angles of the wheels, SWOM assumes the singular configuration only when the traveling direction of the wheel is parallel to the rail of the sliding joint because the movement of the wheel is not transmitted to the main body. Each driving unit in the singular configuration constrains the motion of the main body by one DOF. Then SWOM becomes incapable of omnidirectional movement, but it can move in the allowable directions unless all driving units are in the singular configuration at the same time.

\section{Design of control system for SWOM}

In this section, we discuss the construction of a controller for SWOM based on kinematics.

\subsection{Kinematic model}

The kinematic model of SWOM is explained first. Figure 4(a) shows the reference frames used in the analysis. The global reference frame $O-X_{I} Y_{I}$ is fixed to the floor, while the local reference frame $P-X_{R} Y_{R}$ is fixed to the main body of SWOM. The origin of the local reference frame $P$ is centered on the base block, and $X_{R}$ is parallel to one of the sliding joint rails. We assign the driving unit attached to the sliding joint parallel to $X_{R}$ as driving unit 1 , and the others as driving units 2 and 3 counterclockwise. The pose of the local reference frame in the global frame is set as $\left[\begin{array}{lll}x & y\end{array}\right]^{T}$, which represents the position and orientation of the main body. Figure 4(b) shows the parameters of the wheel mechanism. The angle between the sliding joint rail and $X_{R}$ is $\alpha$, the angle between the wheel axle and the rail is $\beta$, the distance between the center of the base block and the wheel is $l$, the rotation angle of the wheel is $\varphi$, and the radius of the wheel is $r$. In the following discussion, the subscript $i$ represents the parameters belonging to driving unit $i$ ( $i=$ 


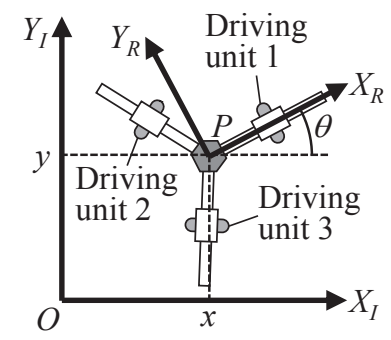

(a)

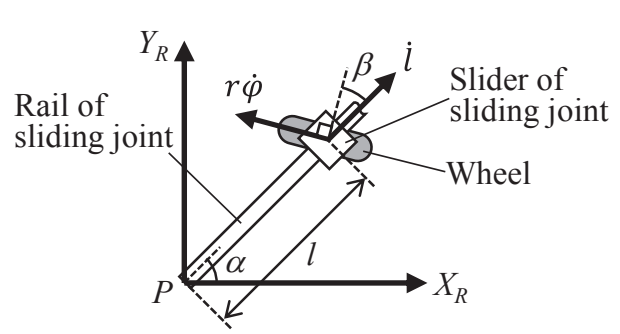

(b)

Fig. 4 Reference frames for analysis of SWOM: (a) Position and orientation of SWOM in the global reference frame. (b) Wheel parameters in the local reference frame.

\section{$1,2,3)$.}

The kinematics of SWOM is described using the parameters defined above. The following kinematic conditions are given with respect to the traveling direction of the wheel and the direction perpendicular to it (Terakawa et al., 2018):

$$
\begin{aligned}
& {\left[\begin{array}{lll}
\sin \left(\alpha_{i}+\beta_{i}\right) & -\cos \left(\alpha_{i}+\beta_{i}\right)-l_{i} \cos \beta_{i}
\end{array}\right] R(\theta)\left[\begin{array}{c}
\dot{x} \\
\dot{y} \\
\dot{\theta}
\end{array}\right]+r \dot{\varphi}_{l}+i_{l} \sin \beta_{i}=0} \\
& {\left[\begin{array}{lll}
\cos \left(\alpha_{i}+\beta_{i}\right) & \sin \left(\alpha_{i}+\beta_{i}\right) & l_{i} \sin \beta_{i}
\end{array}\right] R(\theta)\left[\begin{array}{c}
\dot{x} \\
\dot{y} \\
\dot{\theta}
\end{array}\right]+i_{l} \cos \beta_{i}=0,}
\end{aligned}
$$

where

$$
R(\theta)=\left[\begin{array}{ccc}
\cos \theta & \sin \theta & 0 \\
-\sin \theta & \cos \theta & 0 \\
0 & 0 & 1
\end{array}\right]
$$

is the rotation matrix to transform the vector in the global reference frame to the vector in the local reference frame. Equations (1) and (2) represent the nonholonomic constraints on the movement of the wheels. By focusing on the motion of the main body of SWOM, Eqs. (1) and (2) are always satisfied at an arbitrary velocity $\left[\begin{array}{lll}\dot{x} & \dot{y} & \dot{\theta}\end{array}\right]^{T}$ under the condition $\beta_{i} \neq \pm \pi / 2$ if the rotational velocity of the wheel $\dot{\varphi}_{i}$ and the relative velocity of the sliding joints $\dot{l}_{i}$ can assume an arbitrary value. This theoretically proves the omnidirectional mobility of SWOM. The condition $\beta_{i}= \pm \pi / 2$ corresponds to the singular configuration. Every driving unit in the singular configuration generates the nonholonomic constraint on the motion of the main body and reduces the DOF by one.

The transformation of Eqs. (1) and (2) yields the following equations:

$$
\left[\begin{array}{cc}
A_{1} R & O \\
A_{2} R & I
\end{array}\right] \dot{q}=-\left[\begin{array}{c}
B_{1} \\
B_{2}
\end{array}\right]\left[\begin{array}{c}
\dot{\varphi}_{1} \\
\dot{\varphi_{2}} \\
\dot{\dot{\varphi}_{3}}
\end{array}\right]
$$

where

$$
\begin{aligned}
& q=\left[\begin{array}{llllll}
x & y & \theta & l_{1} & l_{2} & l_{3}
\end{array}\right]^{T} \\
& A_{1}\left(l_{1}, l_{2}, l_{3}\right)=\left[\begin{array}{lll}
\sin \alpha_{1} & -\cos \alpha_{1} & -l_{1} \\
\sin \alpha_{2} & -\cos \alpha_{2} & -l_{2} \\
\sin \alpha_{3} & -\cos \alpha_{3} & -l_{3}
\end{array}\right], B_{1}\left(\beta_{1}, \beta_{2}, \beta_{3}\right)=\left[\begin{array}{ccc}
r \cos \beta_{1} & 0 & 0 \\
0 & r \cos \beta_{2} & 0 \\
0 & 0 & r \cos \beta_{3}
\end{array}\right] \\
& A_{2}=\left[\begin{array}{lll}
\cos \alpha_{1} & \sin \alpha_{1} & 0 \\
\cos \alpha_{2} & \sin \alpha_{2} & 0 \\
\cos \alpha_{3} & \sin \alpha_{3} & 0
\end{array}\right], B_{2}\left(\beta_{1}, \beta_{2}, \beta_{3}\right)=\left[\begin{array}{ccc}
r \sin \beta_{1} & 0 & 0 \\
0 & r \sin \beta_{2} & 0 \\
0 & 0 & r \sin \beta_{3}
\end{array}\right] \text {. }
\end{aligned}
$$

The rotation matrix $R(\theta)$ is always regular, and the matrix $A_{1}\left(l_{1}, l_{2}, l_{3}\right)$ is also regular under the condition $l_{i}>0$. Then, Eq. (4) can be rewritten as 


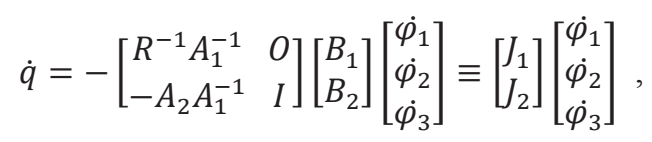

where

$$
\begin{aligned}
& J_{1}\left(\theta, l_{1}, l_{2}, l_{3}, \beta_{1}, \beta_{2}, \beta_{3}\right):=-R^{-1}(\theta) A_{1}^{-1}\left(l_{1}, l_{2}, l_{3}\right) B_{1}\left(\beta_{1}, \beta_{2}, \beta_{3}\right) \\
& J_{2}\left(l_{1}, l_{2}, l_{3}, \beta_{1}, \beta_{2}, \beta_{3}\right):=A_{2} A_{1}^{-1}\left(l_{1}, l_{2}, l_{3}\right) B_{1}\left(\beta_{1}, \beta_{2}, \beta_{3}\right)-B_{2}\left(\beta_{1}, \beta_{2}, \beta_{3}\right) .
\end{aligned}
$$

\subsection{Feedback linearization}

A linearized state equation is derived from the kinematics described in Section 3.1. The time derivative of Eq. (5) is given by

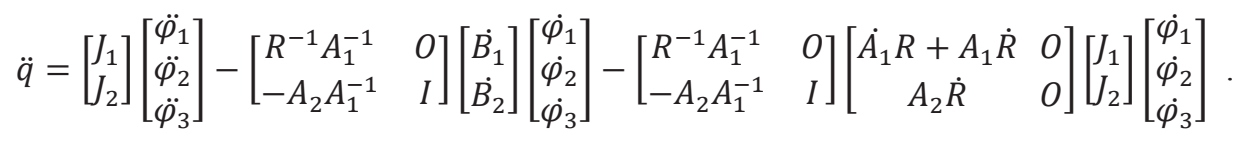

The time derivatives of the matrices in this equation are calculated as follows:

$$
\dot{A_{1}}=-\left[\begin{array}{l}
\dot{l}_{1} \\
\dot{l_{2}} \\
\dot{l_{3}}
\end{array}\right]\left[\begin{array}{lll}
0 & 0 & 1
\end{array}\right]=-J_{2}\left[\begin{array}{c}
\dot{\varphi}_{1} \\
\dot{\varphi_{2}} \\
\dot{\varphi_{3}}
\end{array}\right]\left[\begin{array}{lll}
0 & 0 & 1
\end{array}\right], \dot{B_{1}}=-B_{2} \operatorname{diag}\left(\dot{\beta_{1}}, \dot{\beta_{2}}, \dot{\beta_{3}}\right), \dot{B_{2}}=B_{1} \operatorname{diag}\left(\dot{\beta_{1}}, \dot{\beta_{2}}, \dot{\beta_{3}}\right) .
$$

Then Eq. (6) is arranged as

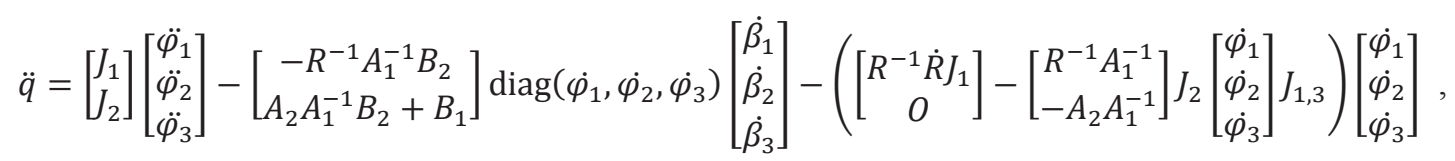

where $J_{1,3}\left(\theta, l_{1}, l_{2}, l_{3}, \beta_{1}, \beta_{2}, \beta_{3}\right)$ is the third row vector of $J_{1}\left(\theta, l_{1}, l_{2}, l_{3}, \beta_{1}, \beta_{2}, \beta_{3}\right)$. Here, we set a part of the state variables of the system as $w=\left[\begin{array}{llllll}\dot{\varphi}_{1} & \dot{\varphi_{2}} & \dot{\varphi}_{3} & \beta_{1} & \beta_{2} & \beta_{3}\end{array}\right]^{T}$ and the input vector to the system as $u=$ $\left[\begin{array}{llllll}\ddot{\varphi_{1}} & \ddot{\varphi_{2}} & \ddot{\varphi_{3}} & \dot{\beta_{1}} & \dot{\beta_{2}} & \dot{\beta}_{3}\end{array}\right]^{T}$. According to Eqs. (1) and (2), $w$ assumes a function of $q$ and $\dot{q}$. As a result, the following equation is given:

$$
\ddot{q}=N(q, w(q, \dot{q})) u+k(q, \dot{q}, w(q, \dot{q})),
$$

where

$$
\begin{aligned}
& N(q, w(q, \dot{q}))=\left[\begin{array}{cc}
J_{1} & R^{-1} A_{1}^{-1} B_{2} \operatorname{diag}\left(\dot{\varphi}_{1}, \dot{\varphi_{2}}, \dot{\varphi_{3}}\right) \\
J_{2} & -\left(A_{2} A_{1}^{-1} B_{2}+B_{1}\right) \operatorname{diag}\left(\dot{\varphi}_{1}, \dot{\dot{\varphi}_{2}}, \dot{\varphi_{3}}\right)
\end{array}\right] \\
& k(q, \dot{q}, w(q, \dot{q}))=-\left(\left[\begin{array}{c}
R^{-1} \dot{R} J_{1} \\
0
\end{array}\right]-\left[\begin{array}{c}
R^{-1} A_{1}^{-1} \\
-A_{2} A_{1}^{-1}
\end{array}\right] J_{2}\left[\begin{array}{c}
\dot{\varphi_{1}} \\
\dot{\dot{\varphi}_{2}} \\
\dot{\varphi_{3}}
\end{array}\right] J_{1,3}\right)\left[\begin{array}{c}
\dot{\dot{\varphi}_{1}} \\
\dot{\dot{\varphi}_{2}} \\
\dot{\varphi_{3}}
\end{array}\right] .
\end{aligned}
$$

Next, we introduce a new input vector $v$ satisfying the following condition:

$$
u=N^{-1}(q, w(q, \dot{q}))\{v-k(q, \dot{q}, w(q, \dot{q}))\} .
$$

Using this input, Eq. (9) is transformed into

$$
\ddot{q}=v \text {. }
$$

When we define $\xi=\left[\begin{array}{ll}q & \dot{q}\end{array}\right]^{T}$, the linearized state equation with the state $\xi$ and the input $v$ is given as follows:

$$
\dot{\xi}=C \xi+D v,
$$

where 


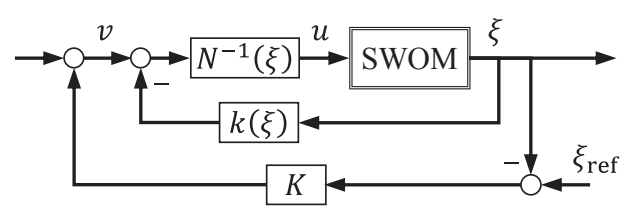

Fig. 5 Structure of the feedback controller for SWOM

$$
C=\left[\begin{array}{ll}
O & I \\
O & O
\end{array}\right], D=\left[\begin{array}{l}
O \\
I
\end{array}\right]
$$

This derives the exactly linearized state equation for SWOM.

Finally, the controller stabilizing the system described by Eq. (12) is designed. When the desired value of the state $\xi$ is expressed as $\xi_{\text {ref }}$, the state feedback is constructed as follows:

$$
v=-K\left(\xi-\xi_{\text {ref }}\right),
$$

where $K \in \mathbb{R}^{6 \times 12}$ is the feedback gain. The system is stabilized by the feedback gain given by

$$
\begin{aligned}
& K=\left[\operatorname{diag}\left(k_{11}, k_{12}, \cdots, k_{16}\right) \operatorname{diag}\left(k_{21}, k_{22}, \cdots, k_{26}\right)\right] \\
& k_{m n}>0 \quad(m=1,2, n=1,2, \cdots, 6) .
\end{aligned}
$$

When this linear controller is used, the input to the original system $u$ is calculated by Eqs. (10) and (13).

$$
u=-N^{-1}(\xi)\left\{K\left(\xi-\xi_{\text {ref }}\right)+k(\xi)\right\} \text {. }
$$

The overall structure of the constructed control system is shown in Fig. 5. We call this feedback controller the normal controller in the following discussions.

\subsection{Avoiding singular configurations}

In Eq. (15), $N^{-1}(\xi)$ is calculated as follows:

$$
N^{-1}(\xi)=-\left[\begin{array}{ccccccc}
\frac{\sin \left(\alpha_{1}+\beta_{1}+\theta\right)}{r} & -\frac{\cos \left(\alpha_{1}+\beta_{1}+\theta\right)}{r} & -\frac{l_{1} \cos \beta_{1}}{r} & \frac{\sin \beta_{1}}{r} & 0 & 0 \\
\frac{\sin \left(\alpha_{2}+\beta_{2}+\theta\right)}{r} & -\frac{\cos \left(\alpha_{2}+\beta_{2}+\theta\right)}{r} & -\frac{l_{2} \cos \beta_{2}}{r} & 0 & \frac{\sin \beta_{2}}{r} & 0 \\
\frac{\sin \left(\alpha_{3}+\beta_{3}+\theta\right)}{r} & -\frac{\cos \left(\alpha_{3}+\beta_{3}+\theta\right)}{r} & -\frac{l_{3} \cos \beta_{3}}{r} & 0 & 0 & \frac{\sin \beta_{3}}{r} \\
\frac{\cos \left(\alpha_{1}+\beta_{1}+\theta\right)}{r \dot{\varphi}_{1}} & \frac{\sin \left(\alpha_{1}+\beta_{1}+\theta\right)}{r \dot{\varphi}_{1}} & \frac{l_{1} \sin \beta_{1}}{r \dot{\varphi}_{1}} & \frac{\cos \beta_{1}}{r \dot{\varphi}_{1}} & 0 & 0 \\
\frac{\cos \left(\alpha_{2}+\beta_{2}+\theta\right)}{r \dot{\varphi}_{2}} & \frac{\sin \left(\alpha_{2}+\beta_{2}+\theta\right)}{r \dot{\varphi}_{2}} & \frac{l_{2} \sin \beta_{2}}{r \dot{\varphi}_{2}} & 0 & \frac{\cos \beta_{2}}{r \dot{\varphi}_{2}} & 0 \\
\frac{\cos \left(\alpha_{3}+\beta_{3}+\theta\right)}{r \dot{\varphi}_{3}} & \frac{\sin \left(\alpha_{3}+\beta_{3}+\theta\right)}{r \dot{\varphi}_{3}} & \frac{l_{3} \sin \beta_{3}}{r \dot{\varphi}_{3}} & 0 & 0 & \frac{\cos \beta_{3}}{r \dot{\varphi}_{3}}
\end{array}\right] .
$$

Focusing on the lower three rows of $N^{-1}(\xi), N(\xi)$ becomes singular for $\dot{\varphi}_{\iota}=0$ and the feedback input $\dot{\beta}_{\imath}$ diverges according to Eqs. (15) and (16). However, the feedback input $\ddot{\varphi}_{l}$, which is determined by the upper three rows of $N^{-1}(\xi)$, never diverges, so that at least three-DOF motion can be controlled even when the configuration is singular as $\dot{\varphi}_{\iota}=0$. Thus, we propose a strategy to control the position and orientation of the main body with priority by switching the feedback controller to another one neglecting the state values $l_{i}$ temporarily near $\dot{\varphi}_{l}=0$. The concrete form of the switching controller is described below.

We set $q_{1}=\left[\begin{array}{lll}x & y & \theta\end{array}\right]^{T}, u_{1}=\left[\begin{array}{lll}\ddot{\varphi}_{1} & \ddot{\varphi}_{2} & \ddot{\varphi}_{3}\end{array}\right]^{T}$, and $u_{2}=\left[\begin{array}{lll}\dot{\beta}_{1} & \dot{\beta}_{2} & \dot{\beta}_{3}\end{array}\right]^{T}$ and extract the first to third rows of Eq. (9).

$$
\ddot{q}_{1}=J_{1}(\xi) u_{1}+R^{-1}(\xi) A_{1}^{-1}(\xi) B_{2}(\xi) \operatorname{diag}\left(\dot{\varphi}_{1}, \dot{\varphi}_{2}, \dot{\varphi}_{3}\right) u_{2}+k(\xi) .
$$

Here, we consider that $u_{2}$ is controlled independently from $u_{1}$ and give a new input vector $v_{1}^{\prime}$ for $u_{1}$ to satisfy the following condition:

$$
u_{1}=J_{1}^{-1}(\xi)\left\{v_{1}^{\prime}-k(\xi)-R^{-1}(\xi) A_{1}^{-1}(\xi) B_{2}(\xi) \operatorname{diag}\left(\dot{\varphi}_{1}, \dot{\varphi}_{2}, \dot{\varphi}_{3}\right) u_{2}\right\}
$$


Then, the input-output relation is given as follows:

$$
\ddot{q}_{1}=v_{1}^{\prime}
$$

This is the same form as Eq. (10), so that the linearized state equation and the stabilizing feedback input are derived as well. Specifically, the feedback input is

$$
v_{1}^{\prime}=-K_{1}^{\prime}\left(\left[\begin{array}{l}
q_{1} \\
\dot{q}_{1}
\end{array}\right]-\left[\begin{array}{l}
q_{1 \mathrm{ref}} \\
\dot{q}_{1 \mathrm{ref}}
\end{array}\right]\right)
$$

where $K_{1}^{\prime} \in \mathbb{R}^{3 \times 6}$ is the feedback gain and $q_{1 \text { ref }}$ and $\dot{q}_{1 \text { ref }}$ are the desired value of $q_{1}$ and $\dot{q}_{1}$, respectively. Here, the condition that $J_{1}(\xi)$ does not have an inverse matrix in Eq. (18) is given as $\beta_{i}= \pm \pi / 2$. This corresponds to the singular configuration where the traveling direction of the wheel is parallel to the sliding joint rail, as explained in Sections 2 and 3.1. However, $\beta_{i}$ and $\dot{\beta}_{\imath}$ are determined arbitrarily here. Therefore, SWOM can avoid the singular configuration $\beta_{i}= \pm \pi / 2$ by using the feedback to make $\beta_{i} \rightarrow n_{i} \pi\left(n_{i} \in \mathbb{Z}\right)$, for example,

$$
u_{2}=-K_{2}^{\prime}\left[\begin{array}{l}
\beta_{1}-n_{1} \pi \\
\beta_{2}-n_{2} \pi \\
\beta_{3}-n_{3} \pi
\end{array}\right]
$$

where $K_{2}^{\prime} \in \mathbb{R}^{3 \times 3}$ is the feedback gain. We call this feedback controller the secondary controller.

Here we summarize the control strategy explained in this section. Away from the singular configuration $\dot{\varphi}_{l}=0$, all of the state variables of SWOM, including $l_{i}$, are controlled using the normal controller constructed in Section 3.2 . Near $\dot{\varphi}_{l}=0$, the controller is switched to the secondary controller proposed in this section, where the position and orientation of the main body are controlled, but $l_{i}$ is temporarily neglected. In this situation, there are concerns that the driving units move out of the range of the sliding joint rails. However, this does not occur if the driving units are placed sufficiently far away from the ends of the sliding joints and the required movement after switching the controller is adjusted within a specified range before switching the controller. In the secondary controller, there exists the singular configuration $\beta_{i}= \pm \pi / 2$, not $\dot{\varphi}_{\iota}=0$, but it is avoidable because $\beta_{i}$ can be controlled to an arbitrary desired value instead of $l_{i}$. Note that in the normal controller, it is unnecessary to avoid $\beta_{i}= \pm \pi / 2$ because the system does not assume a singular configuration except when $\dot{\varphi}_{l}=0$.

\section{Simulation and experiment 4.1 Simulation}

To verify the effectiveness of the control system constructed as described in Section 3, we conducted a simulation. The design parameters were set as follows: $\alpha_{1}=0, \alpha_{2}=2 \pi / 3, \alpha_{3}=4 \pi / 3, r=0.064, k_{11}=k_{12}=0.25, k_{13}=$ $0.16, \quad k_{14}=k_{15}=k_{16}=1.0, \quad k_{21}=k_{22}=1.0, \quad k_{23}=0.80, \quad k_{24}=k_{25}=k_{26}=2.0 \quad, \quad K_{1}^{\prime}=0.75 \times$ $\left[\operatorname{diag}\left(k_{11}, k_{12}, k_{13}\right) \operatorname{diag}\left(k_{21}, k_{22}, k_{23}\right)\right]$, and $K_{2}^{\prime}=\operatorname{diag}(0.20,0.20,0.20)$. The initial state was

$$
\xi=\left[\begin{array}{llllllllllll}
0.00 & 0.00 & 0.00 & 0.20 & 0.20 & 0.20 & 0.00 & 0.00 & 0.00 & 0.00 & 0.00 & 0.00
\end{array}\right]^{T}
$$

and the desired states at times $t=20,40$, and 60 were

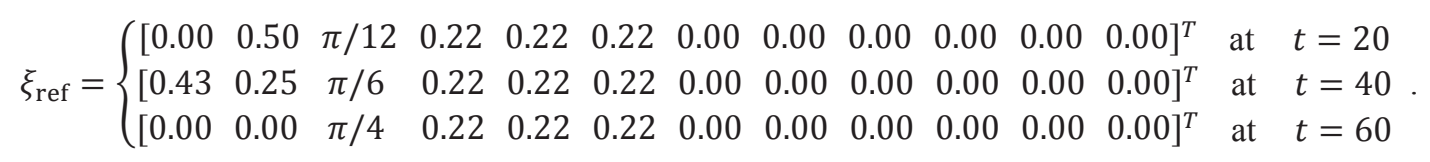

Additionally, the following conditions were given in the simulation. First, the condition of switching the controllers was $\left|\dot{\varphi}_{l}(t)\right|<\pi / 60 \mathrm{~V}\left\|q_{1}(t)-q_{1 \mathrm{ref}}\right\|<0.035$. The former condition defines the vicinity of the singular configuration $\dot{\varphi}_{l}=0$, while the latter condition restricts the movement after switching the controller within a sufficiently small range. Next, the limits of the rotational speed and acceleration of the driving and steering motors were set as

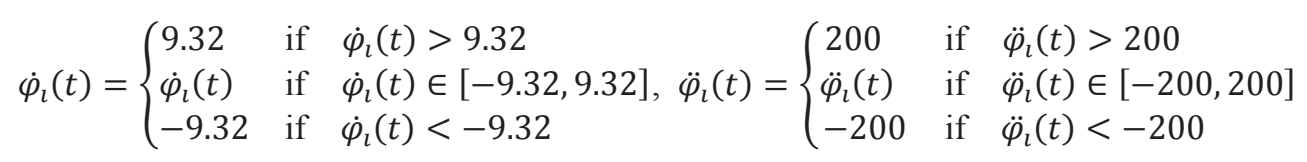



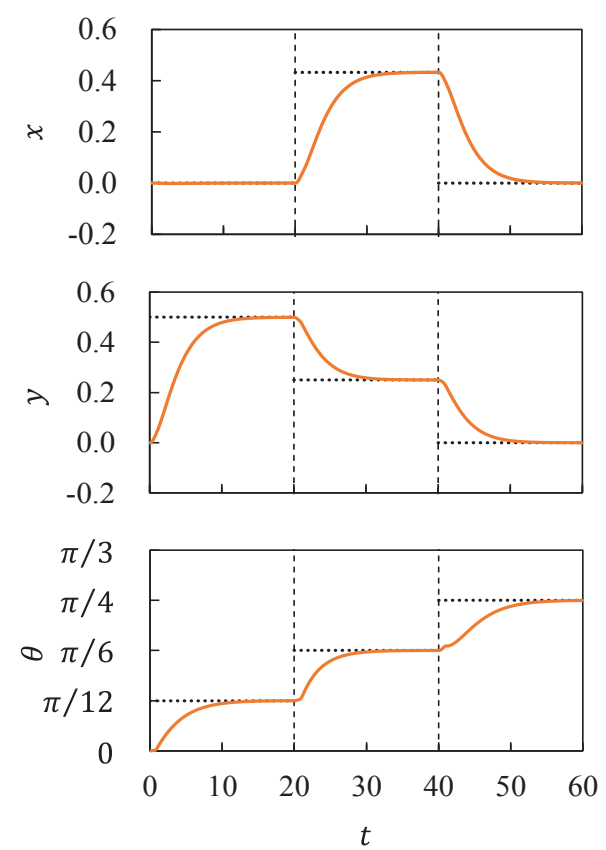

(a)
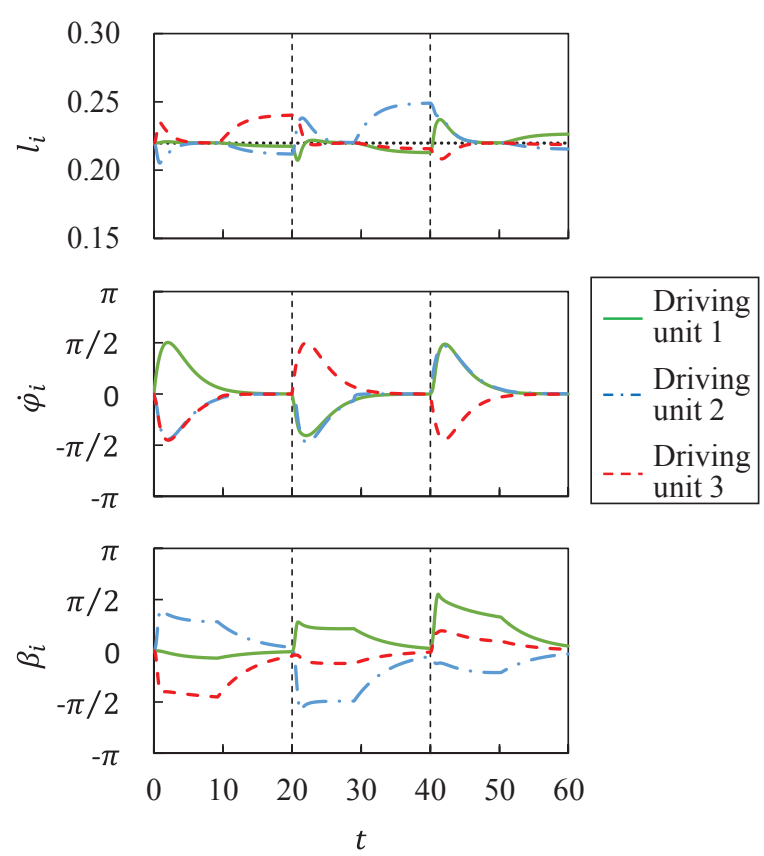

(b)

Fig. 6 Simulation results: (a) Trajectory of the main body of SWOM. The dotted line indicates the desired value. (b) Time variation of the parameters of each driving unit—relative position $l_{i}$, wheel rotational speed $\dot{\varphi}_{l}$, and steering angle $\beta_{i}$.

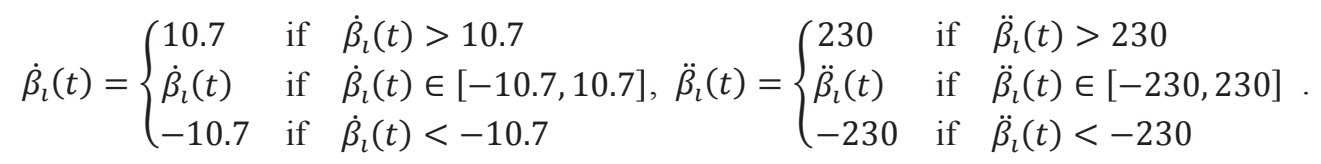

We verify in the simulation that SWOM can converge to the desired state without difficulty under these conditions.

The simulation results are shown in Fig. 6. Figure 6(a) is the trajectory of the main body of SWOM, where each parameter reaches the desired value at $t=20,40$, and 60. Figure 6(b) shows the time variation of the other parameters. In the upper graph, $l_{i}$ repeats convergence to the desired value $l_{i}=0.22$ and deviation. This behavior represents the switching of the controller, where the state variables of SWOM, including $l_{i}$, are controlled to the halfway point but $l_{i}$ is not controlled around $t=20,40$, and 60 . However, the values of $l_{i}$ are within $0.20<l_{i}<$ 0.25 for the entire simulation. Therefore, if the movable range of the sliding joints covers $0.20<l_{i}<0.25$, the driving units do not reach the ends of their sliding joints. With respect to $\dot{\varphi}_{\iota}$ and $\beta_{i}$ in the middle and lower graphs, these values varied continuously within a certain range. For $\beta_{i}$ especially, the problem of divergence around the singular configuration $\dot{\varphi}_{l}=0$ did not occur. These results show that the control system worked as expected.

\subsection{Experiment}

To demonstrate the effectiveness of the developed control system, experiments were conducted using the SWOM prototype pictured in Fig. 7. The base block of the prototype is larger than that shown in Fig. 1, but the fundamental structure of the prototype is similar to the structure shown in Fig. 1. Two parallel linear guides placed on the bottom of the main body constitute the sliding joint. The driving unit is composed of the upper and lower parts separated by the linear guides. The traveling direction of the wheel changes by rotating the lower part around the vertical axis. The upper part has a steering motor, and the lower part has a driving motor and a wheel. The output rotation of each motor is transmitted through a gear head and timing belts. The prototype has six ball casters as safety wheels to prevent tipover, but they normally do not affect the motion of the prototype because usually only the three wheels of the driving units contact the ground.

Figure 8 shows the structure of the control system hardware in the SWOM prototype. To measure the state variables for state feedback, we installed motion capture cameras for the position of the main body, linear encoders for 

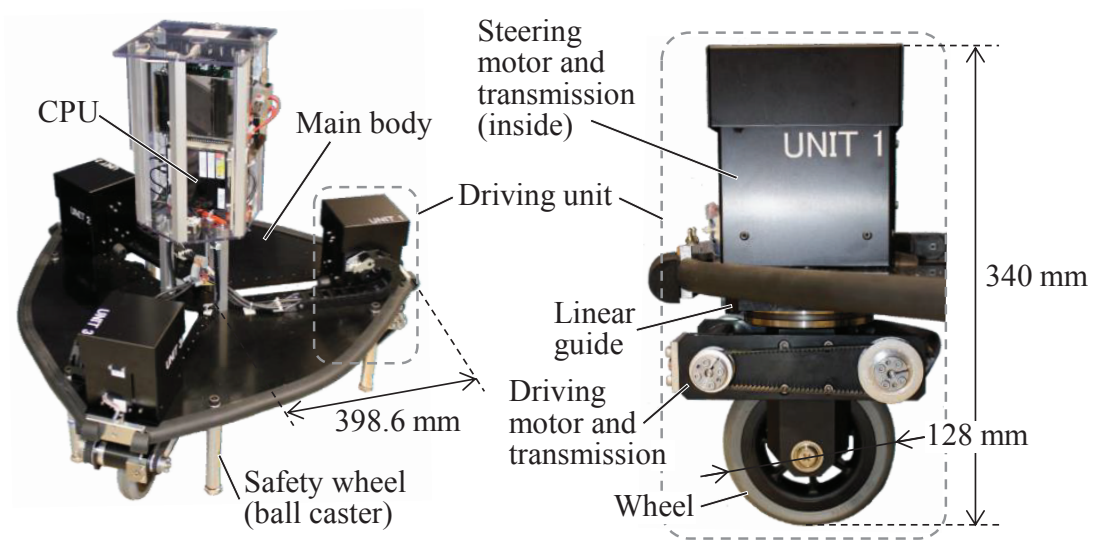

Fig. 7 SWOM prototype

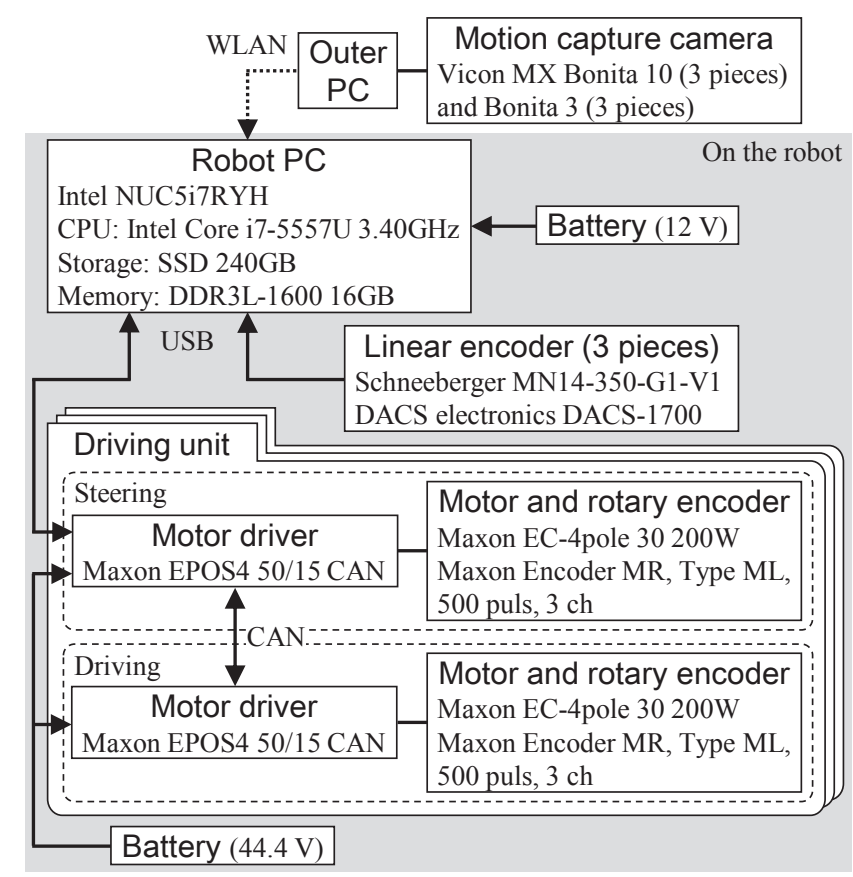

Fig. 8 Control system hardware for SWOM prototype

Table 1 Specifications of SWOM prototype

\begin{tabular}{l|l|l}
\hline \hline Whole system & Weight & $33 \mathrm{~kg}$ \\
\hline \multirow{2}{*}{$\begin{array}{l}\text { Driving unit } \\
\text { (driving / steering) }\end{array}$} & Maximum speed & $9.32 / 10.7 \mathrm{rad} / \mathrm{s}$ \\
\cline { 2 - 3 } & Maximum torque & $38.0 / 41.7 \mathrm{~N} \cdot \mathrm{m}$ \\
\cline { 2 - 3 } & Resolution of encoder & $1.35 \times 10^{-5} / 1.54 \times 10^{-5} \mathrm{rad}$ \\
\hline Linear guide & Movable range & $0.122 \mathrm{~m} \leq l_{i} \leq 0.314 \mathrm{~m}$ \\
\hline Motion capture & Operation period & $1,000 \mathrm{~Hz}$ \\
\hline Linear encoder & Resolution & $0.1 \mu \mathrm{m}$ \\
\hline \hline
\end{tabular}

the displacement of the linear guides, and rotary encoders for the rotating velocity and steering angle/velocity of the wheels. The velocities of the main body and the linear guides were estimated using the complementary filters combining the time difference of each position and the theoretical velocity calculated by Eq. (4). The values measured by the sensors were sent to the PC in the prototype, and after calculation, the PC sent commands to the motors through the motor drivers. The processing was done in a cycle taking about $80 \mathrm{~ms}$. Specifications of the SWOM prototype are shown in Table 1.

We conducted an experiment by making this prototype follow the simulated motion described in Section 4.1. The results are shown in Figs. 9 and 10. Figure 9(a) illustrates the measured path of the SWOM prototype and the simulated 


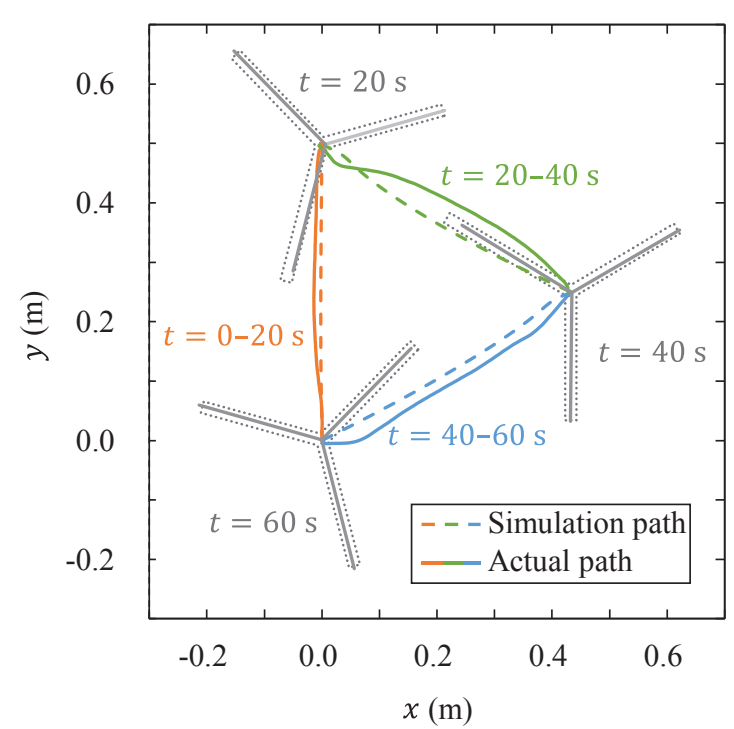

(a)

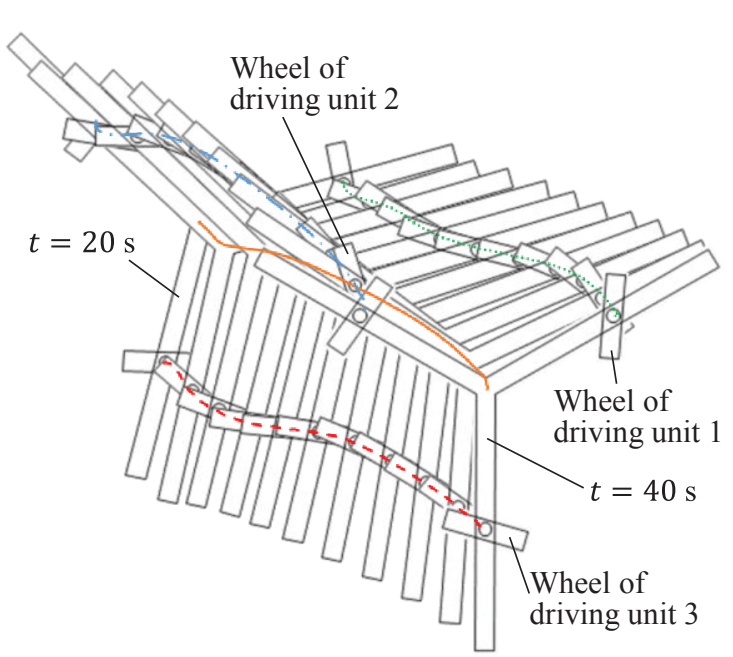

(b)

Fig. 9 (a) Path of the main body of the SWOM prototype. The solid line indicates the actual path of the experimental result measured by the motion capture cameras. The dashed lines indicate the simulation path of the result shown in Fig. 6 . The Y-shaped objects show the states of the SWOM prototype at $t=20,40$, and $60 \mathrm{~s}$, where the solid lines indicates the experimental results and the dotted lines describe the desired states. (b) Motion and paths of the main body and three wheels for $t=20-40 \mathrm{~s}$.
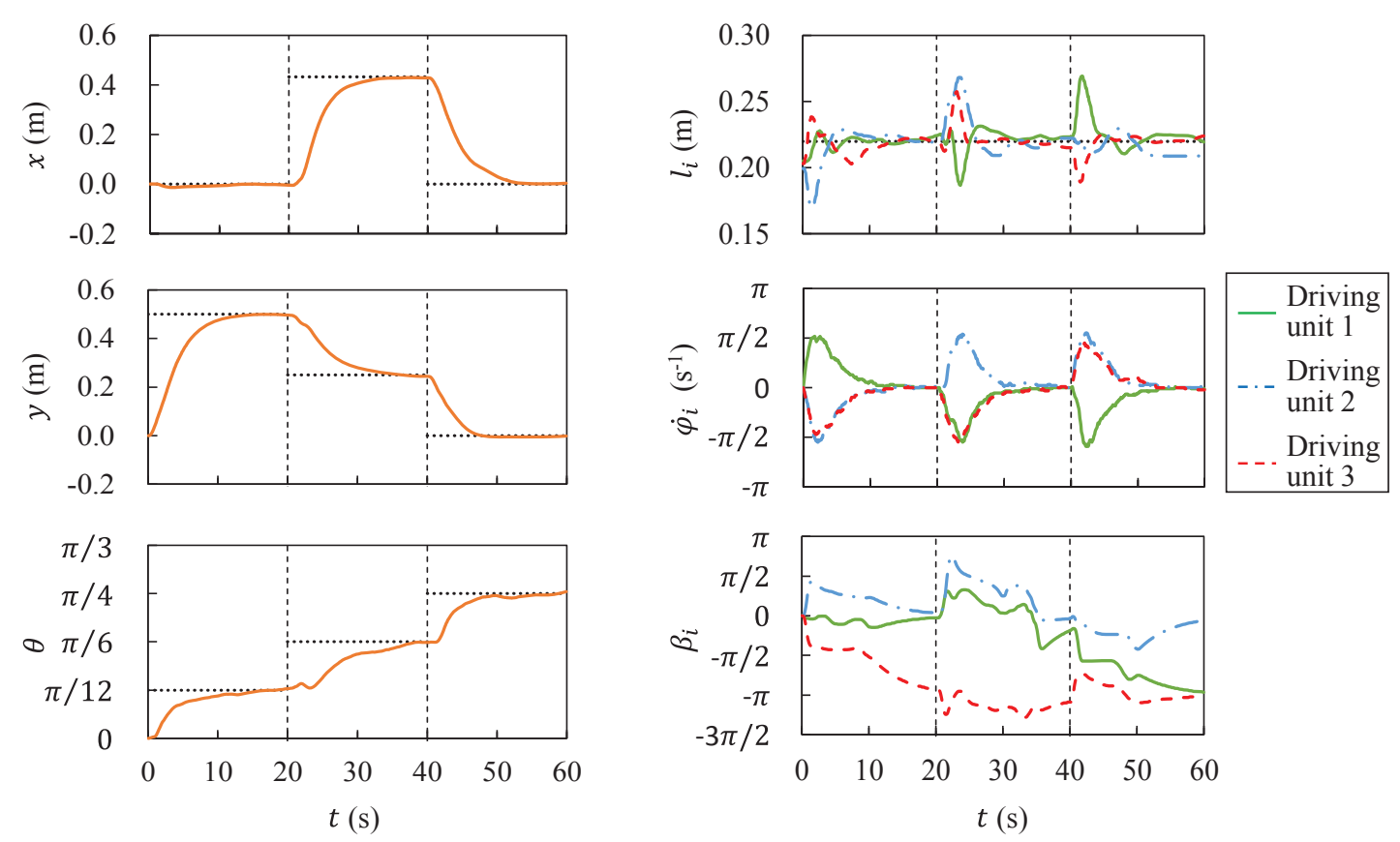

(a)

(b)

Fig. 10 Experimental results: (a) Trajectory of the main body of SWOM given by the motion capture cameras. Dotted lines indicate the desired value. (b) Time variation of the parameters of each driving unit-relative position $l_{i}$, wheel rotational speed $\dot{\varphi}_{l}$, and steering angle $\beta_{i}$ given by the encoders installed in the prototype.

path. To compare the measured state and the desired state at $t=20,40$, and 60 , the position and orientation of the Y-shaped object in the figure indicates the position and orientation of the main body and the length of each limb indicates $l_{i}$ (slider position). The result proves that the prototype reached the desired position and orientation at $t=$ 20,40 , and 60 , though the measured path tended to deviate outward compared to the simulated path. The sequence of the prototype movement for $t=20-40 \mathrm{~s}$ is schematically shown in Fig. 9(b). Each wheel moves along a smooth 
curve while leading the main body to the desired state and keeping the relative position of the sliding joint around the middle of its movable range. Figure 10(a) shows the measured trajectories of the main body, and Fig. 10(b) shows the other state variables. Figure 10(a) demonstrates that the variables $x, y$, and $\theta$ all reach their desired values at $t=20$, 40, and 60. As in the upper plot of Fig. 10(b), $l_{i}$ was maintained within the range where the driving units did not reach the ends of the sliding joint rails. Comparison of $\dot{\varphi}_{l}$ and $\beta_{i}$ in the middle and lower graphs in Fig. 10(b) shows that $\beta_{i}$ did not diverge, even near the singular configuration $\dot{\varphi}_{l}=0$. These results demonstrate that the developed control system works effectively.

\subsection{Discussion}

Comparison of Figs. 10 and 6 shows that the time variation of $\beta_{i}$ in Fig. 10 is larger than that in Fig. 6. For example, $\beta_{3}$ varied over the range of $-\pi / 2 \leq \beta_{3} \leq \pi / 2$ in Fig. 6, whereas $\beta_{3}$ in Fig. 10 existed mainly in the range of $-3 \pi / 2 \leq \beta_{3} \leq-\pi / 2$. This is not because of the divergence of $\beta_{i}$ in the singular configuration $\dot{\varphi}_{\imath}=0$ but rather because of the symmetrical structure of the driving units. In the driving units of SWOM, changing the steering angle of a wheel by $\pm \pi$ is substantially the same as driving the wheel in reverse. Then, we designed the system for $\beta_{i}$ to approach the nearest $n_{i} \pi$ in Eq. (21) regardless of what direction the wheel was traveling. Actually, the values of $\dot{\varphi}_{3}$ in Fig. 10 and Fig. 6 reversed in positivity and negativity for $20 \mathrm{~s} \leq t \leq 60 \mathrm{~s}$, but this phenomenon does not disrupt the motion and control of the SWOM prototype.

When we compare other parameters, the maximum variation of $l_{i}$ from the desired value is larger in Fig. 10 than in Fig. 6. Such $l_{i}$ peaks do not appear during the secondary controller is applied where $l_{i}$ is not controlled but during the normal controller is applied where the state variables, including $l_{i}$, are controlled. This suggests that some difference between the SWOM prototype and the model used in the control system affects its stability. Considering that the developed control system is based completely on kinematics, the error factors are thought to come mainly from dynamical disturbances, such as wheel slippage, sliding joint friction, and drive mechanism viscosity. We can address these factors and achieve a more stable system using a disturbance observer or a dynamics-based controller. Additionally, the proposed control system handles the condition that the driving units do not get to the ends of the sliding joints, that is, $l_{i}$ is limited within a certain range, by giving large feedback gains and making $l_{i}$ converge as quickly as possible when the normal controller is applied. However, this may decrease the efficiency of movement because $l_{i}$ can have priority over the position and orientation of the main body in the control system. Moreover, this control method does not assure that $l_{i}$ values stay away from the upper and lower limits. Regarding this issue, we can use a feedback controller based on the inequality constraints of $l_{i}$, such as a potential method.

\section{Conclusion}

Omnidirectional mobile robots are expected to work efficiently in factories and warehouses because of their superb mobility. However, omnidirectional mobile robots proposed previously have problems, such as low durability, due to the complex structure of the wheel. To solve this problem, we proposed the omnidirectional mobile robot named SWOM, which connect the wheels to the main body with passive sliding joints. SWOM can achieve omnidirectional movement using only conventional wheels to realize both superb mobility and a simple structure.

Based on the kinematic equations of SWOM, which is described as a nonlinear system with nonholonomic constraints, this paper derives the exactly linearized state equation of SWOM using feedback and coordinate transformation to design a controller that stabilizes the linearized system. The unwanted singular configurations in the controller were evaluated and a control strategy to avoid them was proposed. Numerical simulations and experiments demonstrated that SWOM moved as expected and verified the effectiveness of the developed control system.

\section{Acknowledgment}

This work was supported by the Japan Society for the Promotion of Science, JSPS Kakenhi Grant Number JP18J13377. 


\section{References}

Bischoff, R., Huggenberger, U. and Prassler, E., KUKA youBot - a mobile manipulator for research and education, Proceedings of the 2011 IEEE International Conference on Robotics and Automation (ICRA) (2011), pp.1-4.

Campion, G. and Bastin, G., On adaptive linearizing control of omnidirectional mobile robots, Proceedings of the 1989 International Symposium on the Mathematical Theory of Networks and Systems (MTNS-89) (1990), Vol. 2, pp. 531-538.

Cardarelli, E., Digani, V., Sabattini, L., Secchi, C. and Fantuzzi, C., Cooperative cloud robotics architecture for the coordination of multi-AGV systems in industrial warehouses, Mechatronics, Vol. 45 (2017), pp.1-13.

Causo, A., Chong, Z. H., Luxman, R. and Chen, I. M., Visual marker-guided mobile robot solution for automated item picking in a warehouse, Proceedings of the 2017 IEEE International Conference on Advanced Intelligent Mechatronics (AIM) (2017), pp.201-206.

d'Andrea-Novel, B., Bastin, G. and Campion, G., Dynamic feedback linearization of nonholonomic wheeled mobile robots, Proceedings of the 1992 IEEE International Conference on Robotics and Automation (ICRA) (1992), pp.2527-2532.

d'Andrea-Novel, B., Bastin, G. and Campion, G., Control of nonholonomic wheeled mobile robots by state feedback linearization, The International Journal of Robotics Research, Vol. 14, No. 6 (1995), pp.543-559.

de Best, J., van de Molengraft, R. and Steinbuch, M., A novel ball handling mechanism for the RoboCup middle size league, Mechatronics, Vol. 21, No. 2 (2011), pp.469-478.

Ferrière, L., Rauccent, B. and Campion, G., Design of omnimobile robot wheels, Proceedings of the 1996 IEEE International Conference on Robotics and Automation (ICRA) (1996), pp.3664-3670.

Gross, H. M., Scheidig, A., Debes, K., Einhorn, E., Eisenbach, M., Mueller, S., Schmiedel, T., Trinh, T. Q., Weinrich, C., Wengefeld, T., Bley, A. and Martin, C., ROREAS: robot coach for walking and orientation training in clinical post-stroke rehabilitation-prototype implementation and evaluation in field trials, Autonomous Robots, Vol. 41, No. 3 (2017), pp.678-698.

Guo, S., Jin, Y., Bao, S. and Xi, F. F., Accuracy analysis of omnidirectional mobile manipulator with mecanum wheels, Advances in Manufacturing, Vol. 4, No. 4 (2016), pp.363-370.

Komori, M., Matsuda, K., Terakawa, T., Takeoka, F., Nishihara, H. and Ohashi, H., Active omni wheel capable of active motion in arbitrary direction and omnidirectional vehicle, Journal of Advanced Mechanical Design, Systems, and Manufacturing, Vol. 10, No. 6 (2016), Paper No. JAMDSM0086.

Kumagai, M. and Ochiai, T., Development of a robot balanced on a ball - first report, implementation of the robot and basic control—, Journal of Robotics and Mechatronics, Vol. 22, No. 3 (2010), pp.348-355.

Masuzawa, H., Miura, J. and Oishi S., Development of a Mobile Robot for Harvest Support in Greenhouse Horticulture -Person Following and Mapping_, Proceedings of the 2017 IEEE/SICE International Symposium on System Integration (2017), pp.541-546.

Muir, P. and Neuman, C., Kinematic modeling for feedback control of an omnidirectional wheeled mobile robot, Proceedings of the 1987 IEEE International Conference on Robotics and Automation (1987), pp.1772-1778.

Nielsen, I., Dang, Q. V., Bocewicz, G. and Banaszak, Z., A methodology for implementation of mobile robot in adaptive manufacturing environments, Journal of Intelligent Manufacturing, Vol. 28, No. 5 (2017), pp.1171-1188.

Ok, S., Kodama, A., Matsumura, Y. and Nakamura, Y., $\mathrm{SO}(2)$ and $\mathrm{SO}(3)$, omni-directional personal mobility with link-driven spherical wheels, Proceedings of the 2011 IEEE/RSJ International Conference on Intelligent Robots and Systems (IROS) (2011), pp.268-273.

Ostrovskaya, S., Spiteri, R. J. and Angeles, J., Dynamics of a mobile robot with three ball-wheels, International Journal of Robotics Research, Vol. 19, No. 4 (2000), pp.383-393.

Saegusa, S., Yasuda, Y., Uratani, Y., Tanaka, E., Makino, T. and Chang, J. Y., Development of a guide-dog robot: leading and recognizing a visually-handicapped person using a LRF, Journal of Advanced Mechanical Design, Systems, and Manufacturing, Vol. 4, No. 1 (2010), pp.194-205.

Samani, H. A., Abdollahi, A., Ostadi, H. and Rad, S. Z., Design and development of a comprehensive omni directional soccer player robot, International Journal of Advanced Robotic Systems, Vol. 1, No. 3 (2004), pp.191-200.

Singh, A., Paigwar, A., Manchukanti, S. T., Saroya, M., Maurya, M. and Chiddarwar, S., Design and implementation of omni-directional spherical modular snake robot (OSMOS), Proceedings of the IEEE International Conference on 
Mechatronics (ICM) (2017), pp.79-84.

Sori, H., Inoue, H., Hatta, H. and Ando, Y., Effect for a paddy weeding robot in wet rice culture, Journal of Robotics and Mechatronics, Vol. 30, No. 2 (2018), pp.198-205.

Tanaka, K., Okamoto, Y., Ishii, H., Kuroiwa, D., Yokoyama, H., Inoue, S., Shi, Q., Okabayashi, S., Sugahara, Y. and Takanishi, A., A study on path planning for small mobile robot to move in forest area, Proceedings of the 2017 IEEE International Conference on Robotics and Biomimetics (2017), pp.2167-2172.

Terakawa, T., Komori, M., Matsuda, K. and Mikami, S., A novel omnidirectional mobile robot with wheels connected by passive sliding joints, IEEE/ASME Transactions on Mechatronics, Vol. 23, No. 4 (2018), pp. 1716-1727.

Tobita, K., Sagayama, K., Mori, M. and Tabuchi, A., Structure and examination of the guidance robot LIGHBOT for visually impaired and elderly people, Journal of Robotics and Mechatronics, Vol. 30, No. 1 (2018), pp.86-92.

Wada, M. and Asada, H., Design and control of a variable footprint mechanism for holonomic omnidirectional vehicles and its application to wheelchairs, IEEE Transactions on Robotics and Automation, Vol. 15, No. 6 (1999), pp.978-989.

West, M. and Asada, H., Design and control of ball wheel omnidirectional vehicles, Proceedings of 1995 IEEE International Conference on Robotics and Automation (ICRA) (1995), pp.1931-1938. 